receptors and shown to be susceptible to stress. METHODS/STUDY POPULATION: This study explored behavioral and neural impacts of early life stress in Long-Evans rats reared with or without limited access to bedding material during postnatal day (PND) 2-9. Corticosterone (CORT) levels were measured at PND8 and 70. During PND50-70, rats were assessed on Novel Object Recognition to test memory, Rotarod to evaluate cerebellar integrity, Elevated Plus Maze to assay anxiety, Social Preference, and Eyeblink Conditioning, a cerebellar-dependent and endocannabinoid-mediated task. Lipid analysis was performed on PND70 tissue samples of cerebellar interpositus (IP) nucleus via high-performance liquid chromatography and tandem mass spectrometry. RESULTS/ANTICIPATED RESULTS: Both male and female rats experiencing early life stress exhibited significantly impaired recognition memory $(\mathrm{N}=16-20$ / group). Female rats having undergone stress exhibited decreased social preference compared to normally reared females $(\mathrm{N}=11$ / group). Stressed males showed facilitated eyblink conditioning compared to normally reared males $(\mathrm{N}=7-9 /$ group $)$. There were no group differences in rotarod or elevated plus maze performance or CORT levels at PND8 or 70 across rearing groups. At PND70, male rats experiencing early life stress exhibited a significant decrease in 2 -arachidonoyl glycerol (2-AG) and arachidonic acid levels in the IP nucleus compared to normally reared males ( $\mathrm{N}=8-9 /$ group). Compared to normally reared females, those experiencing early life stress exhibited a significant increase in prostaglandin E2 levels in the IP nucleus ( $\mathrm{N}=6-7 /$ group). DISCUSSION/SIGNIFICANCE OF IMPACT: Early life stress, induced by limited bedding, resulted in sex-specific behavioral and lipid impairments. Results suggest that stress causes long-term alterations in endocannabinoid dynamics in males in the cerebellar IP nucleus and sex-related lipids in female cerebellum. These changes may contribute to observed long-term behavioral aberrations. Moreover, findings suggest these behavioral changes may be the result of negative-feedback dysfunction (as evidenced by decreased endocannabinoids in males) or increased neural inflammation or proliferation (as evidenced by increased prostaglandins in females). Future analysis will quantify mRNA and protein for cannabinoid receptors to better characterize aberrations to this system. Moreover, other neural regions dense with cannabinoid receptors (i.e., PFC, hippocampus) will be investigated. This work provides a basis for understanding stress impacts on the development of cognitive deficits observed in psychotic and anxiety disorders. Specifically, facilitation of eyblink conditioning complements research in humans with anxiety disorders. Broadly, understanding stressrelated endocannabinoid dysregulation may provide insights into risks for, and the development of, psychopathology and uncover novel therapeutic targets with high translational power.

\section{Effects of Local Interleukin-6 on Mitochondrial Physiology in Skeletal Muscle}

Hinnah $\mathrm{Abid}^{1}$, Corey Hart and Ian Lanza

${ }^{1}$ Mayo Graduate School

OBJECTIVES/SPECIFIC AIMS: In the context of skeletal muscle, IL-6 plays a major role in muscle quality. The goal of this project was to study the influence of systemic IL-6 on skeletal muscle mitochondrial physiology, most notably mitochondrial function (respiration and ROS production) and mitochondrial content. METHODS/ STUDY POPULATION: To determine the influence of interleukin-6 (IL-6) on skeletal muscle mitochondria, high-resolution respirometry was performed to simultaneously measure oxygen consumption
(JO2) and ROS production in differentiated myotubes incubated with increasing IL-6 $(0,10,50,100 \mathrm{ng} / \mathrm{mL})$ for 18 hours in serum free conditions. To evaluate the impact of IL- 6 on mitochondrial content we performed western blots on cell lysates from treated cells, measuring proteins of the mitochondrial electron transport chain (ETC) using a cocktail antibody and PGC- $1 \alpha /$ PGC- 13 for mitochondrial biogenesis. To determine the role of mitochondrial ROS production on JO2 and mitochondrial content, we co-treated differentiated myotubes for 18 hours with 50 and 100ng/mL IL- 6 and the mitochondrial specific antioxidant, MitoQ and performed respirometry for mitochondrial functional measurements and western blots for mitochondrial content.Statistical significance was evaluated by using a 2-tailed Student's t-test and two-way ANOVA. Post hoc all-group analyses were conducted to determine which groups were different when the model was significant. RESULTS/ANTICIPATED RESULTS: Mitochondrial functional measurements show increased JO2 and increased ROS production in an IL-6 dose-dependent manner. Targeting mitochondrial ROS production with $0.5 \mu \mathrm{m}$ MitoQ attenuated IL-6 induced increases in JO2 and ROS production. Complexes I and II (CI, CII) of the ETC increased significantly in an IL-6 dosewise fashion, and co-treatment with MitoQ normalized increases at $100 \mathrm{ng} / \mathrm{mL}$ Il-6. 100ng/mL IL-6 significantly increased protein expression of PGC- $1 \alpha$ and PGC-1ß. Co-treatment with MitoQ normalized IL-6 induced increase in PGC- $1 \alpha$. DISCUSSION/SIGNIFICANCE OF IMPACT: Our data suggest that when treated chronically at a high dose, IL-6 increases mitochondrial respiration, ROS production, and content. Targeting mitochondrial ROS production normalizes these mitochondrial adaptations. The present study provides new insights into mitochondrial physiology in the context of inflammation. Therapeutically targeting mitochondrial ROS production may impact skeletal muscle quality in certain populations.

3321

\section{European Ancestry as a Risk Factor for Atrial Fibrillation in Puerto Rican Hispanics}

Ariel Gonzalez-Cordero ${ }^{1}$, Jorge Duconge-Soler ${ }^{1}$ and

Ángel López-Candales ${ }^{1}$

${ }^{1}$ University of Puerto Rico-Medical Sciences Campus

OBJECTIVES/SPECIFIC AIMS: Consequently, we have decided to evaluate the presence of single nucleotide polymorphism (SNP) previously associated with AF on a European-descent population in an attempt to first identify the most common loci present in the PRH population and then search for specific PRH SNP associated with AF. METHODS/STUDY POPULATION: A secondary analysis of a Puerto Rican population sample $(n=120)$ from The Pharmacogenetics of Warfarin in Puerto Ricans Study will be performed. We will implement data from the 1000 genome project to establish a control group of healthy PRH population. Will evaluate the presence of 111 known single nucleotide polymorphisms associated with AF in Europeans and determine the frequency in PRH population sample, and validate predictability of such SNPs. Using admixture informatic markers (AIM) analysis will determine the percentage of admixture by Yoruba, Native American and Iberic-European. Statistical analysis will include the use of the Pearson Product-Moment Coefficient correlation analysis and multivariate linear regression. For admixture will use Maximum Likelihood Estimation and Markov Chain Monte Carlo models. RESULTS/ANTICIPATED RESULTS: A higher frequency of AF associated European single nucleotide polymorphisms, and an overall higher percentage of European admixture will be associated with atrial fibrillation in Puerto Rican Hispanic patients. 